\title{
Mechanism of Decreased Forward Stroke Volume in Children and Swine with Ventricular Septal Defect and Failure to Thrive
}

\author{
William J. Corin, " M. Michael Swindle," James F. Spann, Jr.," Kiyoharu Nakano, * Mary Frankis," Robert W. W. Biederman," \\ Alison Smith, ${ }^{\ddagger}$ Ashby Taylor, ${ }^{5}$ and Blase A. Carabello* \\ ${ }^{*}$ Division of Cardiology and Gazes Cardiac Research Institute, ${ }^{\ddagger}$ Department of Comparative Medicine, and ${ }^{\S}$ Division \\ of Pediatric Cardiology, Medical University of South Carolina, Charleston, South Carolina 29425
}

\begin{abstract}
Children with ventricular septal defect (VSD) often demonstrate failure to thrive (FTT). Such patients usually have reduced systemic cardiac output which has been postulated as a cause for their growth retardation. This study was conducted to ascertain the mechanism of the reduced cardiac output in children with VSD and FTT and also in a porcine model of VSD. Forward stroke volume was reduced in VSD-FTT children, $31 \pm 8 \mathrm{ml} / \mathrm{m}^{2}$, compared to normal children, $49 \pm 15 \mathrm{ml} / \mathrm{m}^{2}(P$ $<0.05)$, but was not reduced in children with VSD and normal growth and development $\left(41 \pm 16 \mathrm{ml} / \mathrm{m}^{2}\right)$. Forward stroke volume was also reduced in swine with VSD compared to controls. Contractility assessed by mean velocity of circumferential shortening $\left(V_{\mathrm{ef}}\right)$ corrected for afterload was similar in normals and VSD-FTT children. Contractile performance was also similar in normal and VSD swine. Afterload assessed as systolic stress was similar in FTT-VSD children and normal subjects. Preload assessed as end-diastolic stress was increased in the VSD-FTT group. End-diastolic volume was not larger in the VSD-FTT group. We conclude that the reduced stroke volume seen in VSD-FTT children and VSD-swine was not due to reduced contractility, increased afterload or reduced preload. The reduced stroke volume may have been due to failure of end-diastolic volume to increase adequately.
\end{abstract}

\section{Introduction}

Children with ventricular septal defect (VSD) ${ }^{1}$ often demonstrate failure to thrive (FTT) (1-3). While several mechanisms have been proposed to explain this phenomenon (4-10), the observation that patients with the largest intracardiac shunts demonstrate the most growth retardation suggests a cardiac cause (8-9). Successful correction of the shunt leads to improved growth and development further supporting a cardiac etiology of FTT (11-15). One proposed mechanism is that

Address reprint requests to Dr. Carabello, Division of Cardiology, Medical University of South Carolina, 171 Ashley Avenue, Charleston, SC 29425.

Received for publication 29 July 1987 and in revised form 14 March 1988.

1. Abbreviations used in this paper: Emax, peak elastance; FTT, failure to thrive; NGD, normal growth and development; $S V_{t}$, ventricular strike volume; $V_{\mathrm{cf}}$, velocity of circumferential fiber shortening; VSD, ventricular septal defect.

J. Clin. Invest.

(C) The American Society for Clinical Investigation, Inc. 0021-9738/88/08/0544/08 \$2.00

Volume 82, August 1988, 544-551 reduced systemic cardiac output produces decreased tissue perfusion which results in slowed growth and development (6, 8). Reduced cardiac output could be caused by impaired contractile function, increased afterload, reduced preload or an inadequate compensatory increase in left ventricular end-diastolic volume. The current study was performed to investigate which of these mechanisms reduced systemic cardiac output in children with VSD and FTT. To do this, we compared cardiac mechanics and contractile function at cardiac catheterization in children with VSD and FTT to children with VSD and normal growth and development (NGD), and to normal subjects.

Because obvious ethical constraints limit investigation in potentially unstable children with congenital heart disease, we also studied a newly discovered naturally occurring porcine model of VSD in which systemic stroke volume was reduced. Study in an animal model permitted the use of load manipulation and beta blockade allowing a more precise evaluation of contractile function without the confounding influence of autonomic reflexes.

\section{Methods}

\section{Patient studies}

Study design and patient selection. Hemodynamic and cardiac mechanics data were obtained at cardiac catheterization in 12 children with ventricular septal defect who demonstrated failure to thrive (group VSD-FTT), in 9 children with VSD who demonstrated normal growth and development (group VSD-NGD), and in 11 normal children (group NL). A child was considered to have FTT if his weight was $\leq$ the third percentile for children of that age and sex ( $>-2$ standard deviations from the mean) (16). Normal growth and development was defined as achievement of $\geq$ the sixteenth percentile for children of the same sex and age ( $<-1$ standard deviation from the mean). Normal subjects underwent cardiac catheterization before electrophysiologic testing for cardiac arrhythmias. Children in whom catheterization demonstrated no anatomic or physiologic cardiac abnormalities other than arrhythmias, who were not taking cardiac medications and who were in normal sinus rhythm at the time of study were selected to be normal subjects. These subjects all exhibited normal growth and development. The age and weight of the subjects studied is shown in Table $I$. Analysis of the patients' records demonstrated that when VSD-NGD patients were of a similar age $(1.65 \pm 0.8 \mathrm{yr})$ to the VSD-FTT children, the VSD-NGD patients still demonstrated NGD, average weight percentile $40 \pm 15$.

Catheterization reports for procedures performed from 1 January 1984 to 1 January 1987 were screened to identify patients with isolated ventricular septal defects and to identify normal subjects. 49 subjects with isolated VSD and 22 normal subjects were identified. Patients were then included for study if they met the following criteria: $(a)$ The ratio of pulmonary to systemic blood flow for the patients with VSD was at least 1.75 to 1 . (b) Adequate ventriculograms were available for analysis. An adequate ventriculogram was one in which diastolic, systolic, and wall thickness silhouettes were clearly discernable and in 
Table I. Age and Weight of Patients

\begin{tabular}{lccc}
\hline & Controls & vSD-NGD & vSD-FTT \\
\hline Weight $(k g)$ & $22 \pm 12$ & $17 \pm 7$ & $8 \pm 4^{* \ddagger}$ \\
Weight percentile & $53 \pm 27$ & $41 \pm 17$ & $<3^{* \S}$ \\
Age $(y r)$ & $6.3 \pm 3.5$ & $4.5 \pm 3.1$ & $1.9 \pm 1.5^{*}$ \\
\hline
\end{tabular}

${ }^{*} P<0.01$ vs. controls; ${ }^{\ddagger} P<0.05$ vs. VSD-NGD; ${ }^{\S} P<0.01$ vs. VSD-NGD.

Absence of sign indicates no significant difference.

which two consecutive sinus beats were available for analysis. (c) Properly damped and calibrated pressure tracings were available for review. 21 subjects with isolated VSD ( 12 with FTT and 9 with NGD) and 11 normal subjects met these criteria and were chosen for study.

Catheterization procedure. Children were premedicated with 2 $\mathrm{mg} / \mathrm{kg}$ meperidine and $1 \mathrm{mg} / \mathrm{kg}$ promethazine. Pressures were recorded using properly damped, fluid-filled catheters. Hemoglobin oxygen saturation was determined by reflectance oximetry in the aorta, left ventricle, pulmonary artery, right ventricle, right atrium, and venae cavae. The ratio of pulmonary to systemic blood flow was calculated as: $Q P / Q S=L V$ sat $-R A$ sat $/ L V$ sat $-P A$ sat, where $L V$ sat $=\%$ oxygen saturation in the left ventricle, $P A$ sat $=$ oxygen saturation in the pulmonary artery, and $R A$ sat $=$ oxygen saturation in the right atrium.

Ventriculography was performed at 60 frames/s in the $30^{\circ}$ degree right anterior oblique position. Pressure measurements were taken just before ventriculography. Premature and post-premature beats were excluded from analysis.

Measurements and calculations. Total angiographic left ventricular stroke volume was calculated as end-diastolic volume minus end-systolic volume where volume was derived by the area-length method (17). Volumes were indexed by dividing by body surface area. Forward systemic angiographic stroke volume was calculated as total left ventricular stroke volume divided by the shunt ratio. Angiographic systemic cardiac output $=$ angiographic systemic stroke volume $\times$ heart rate.

Wall thickness was measured at end diastole along the mid-portion of the anterior left ventricular wall. The systolic increase in wall thickness was then calculated using the assumption that left ventricular mass remains constant throughout the cardiac cycle (18). Circumferential wall stress was calculated frame by frame using Mirsky's (19) formula. Frame by frame analysis allowed us to measure end-systolic stress, peak systolic stress, and mean systolic stress. Mean-systolic stress was calculated by summing the individual systolic frame stresses and dividing by the total number of frames.

Left ventricular mass, expressed in grams, was calculated after the method of Rackley et al. (20).

Left ventricular contractile function was assessed using the afterload-corrected mean velocity of circumferential fiber shortening method ( $V_{\mathrm{cr}}$-stress) (21-24). This method corrects a preload independent ejection phase index (mean $V_{\mathrm{cf}}$ ) (25) for afterload (wall stress) yielding a relationship which is independent of preload and corrected for afterload, thus reflecting contractile state. $V_{\mathrm{cf}}$ and mean stress were plotted for the 11 normal subjects. We used mean systolic stress since mean $V_{\mathrm{cf}}$ is measured over all of systole. Patients with VSD were then plotted against the $95 \%$ prediction band for the normal data. $V_{\text {cf }}$ was calculated as: $\mathrm{EDD}-\mathrm{ESD} / \mathrm{EDD} \times \mathrm{ET}$, where $\mathrm{EDD}=$ end diastolic left ventricular minor axis, ESD = end-systolic minor axis, and ET $=$ ejection time, calculated as the total number of frames during which shortening occurred $\times 0.0166 \mathrm{~s} /$ frame. This method was used since in VSD patients ejection could have occurred before the upstroke of the aortic pressure tracing and following the dicrotic notch, the usual markers for measuring ejection time.
Patient followup. Patient followup was obtained by review of the patients' hospital charts at the time of their followup clinic visits after cardiac surgery. This followup data was used to ascertain symptomatic status and growth and development following the correction of the VSD.

\section{Animal studies}

Anesthesia. 13 fasted Yucatan micro swine (Charles River Breeding Laboratories, Wilmington, MA) of both sexes, 5 with normal cardiac anatomy, and 8 with a congenital high membranous VSD, were investigated by cardiac catheterization. On the day of study, the animals were placed in a sling, and initially sedated with isoflurane $2 \%$ delivered with $100 \% \mathrm{O}_{2}$. When adequate relaxation had been achieved, the swine were intubated but continued to breathe without assistance. They were attached to a Jackson-Reese non-rebreathing ventilation circuit through which they received isoflurane 2 to $2.75 \%$ and ambient air, without supplemental oxygen. While breathing this mixture, arterial oxygen saturation remained $>90 \%$. Of the inhalational fluorocarbons, isoflurane has the least impact upon inotropic function (26-29). However as isoflurane has been shown to reduce contractility (30), care was taken to utilize the minimum dose necessary to maintain surgical anesthesia and to administer the drug within a narrow dose range. There was no difference in the concentration of isoflurane used in the two groups of swine.

Catheterization. Right and left heart catheterizations were performed under sterile conditions from either the percutaneous approach or by direct exposure of the cervical or femoral vessels. A balloon-tipped flow directed Swan-Ganz catheter was used for right heart catheterization. Left heart catheterization was performed using a 5-F Bermann catheter, which permitted both pressure and oxygen saturation determinations, and with which left ventriculography was performed. A 6-F micromanometry catheter (Millar Instruments, Houston, TX) was introduced into the ascending aorta in order to measure central aortic pressure simultaneously with ventriculography. Load manipulation was accomplished by inflation and deflation of either a 15 - or $18-\mathrm{mm}$ valvuloplasty balloon that had been positioned from the superior into the inferior vena cava. All pressure, oximetry and cardiac output data were obtained prior to ventriculography.

Shunt and cardiac output determinations. Oxygen saturation was determined in duplicate from the pulmonary artery, right ventricle, right atrium, vena cavae, and left ventricle using an OSM2 Hemoximeter (Radiometer A/S, Copenhagen, Denmark). These data were utilized to calculate the shunt ratio $(Q P / Q S)$ as reported under human studies. Blood hemoglobin $(\mathrm{Hb}, \mathrm{g} / \mathrm{dl})$ concentration was measured using the OSM2 Hemoximeter. The systemic cardiac output was calculated from the Fick method as: Cardiac output (liters/minute) $=V O_{2} /\left(A-V O_{2} \Delta\right)$, where $V O_{2}=$ oxygen consumption per minute determined using a Tissot respirometer $\left(\mathrm{ml} \mathrm{O}_{2} / \mathrm{min}\right) ; A-V \mathrm{O}_{2} \Delta=$ the difference between the oxygen content of arterial and venous blood was determined as:

$$
\begin{aligned}
& A-V \mathrm{O}_{2} \Delta\left(\mathrm{ml} \mathrm{O}_{2} / \text { liter }\right) \\
& =H b(\mathrm{~g} / \text { liter }) \cdot(L V-R A) \cdot\left(1.36 \mathrm{ml} \mathrm{O}_{2} / \mathrm{g} \mathrm{Hb}\right),
\end{aligned}
$$

where $H b=$ hemoglobin concentration, and $(L V-R A)=$ oxygen saturation of the left ventricle - oxygen saturation in right atrium. Pulmonary blood flow was calculated as the product of systemic cardiac output and the shunt ratio.

Ventriculography and volume determinations. Left ventriculography was performed in the right anterior oblique $\left(30^{\circ}\right)$ position using 35 $\mathrm{mm}$ film at a rate of 60 frames per second. Left ventricular volume, thickness and mass were calculated as described under human studies. Cardiac output and stroke volume were determined by both the Fick and angiographic methods. Angiographic left ventricular stroke volume $\left(S V_{\mathrm{A}}\right)$ was determined as: $S V_{\mathrm{A}}(\mathrm{ml})=$ End-diastolic volume $(\mathrm{ml})$ - end-systolic volume $(\mathrm{ml})$. Fick total left ventricular stroke volume $\left(S V_{\mathrm{t}}\right)$ was determined as: $S V_{\mathrm{t}}(\mathrm{ml})=($ systemic blood flow + shunt 
blood flow)/heart rate. Fig. 1 demonstrates a close correlation between values obtained using the two techniques.

$30 \mathrm{~min}$ following the baseline ventriculogram, beta adrenergic blockade was achieved and angiography was repeated during which load was manipulated for assessment of contractile function.

Assessment of contractile function. Contractile function was assessed by several methods. We calculated peak elastance (Emax), which has been shown to be relatively independent of preload, account for afterload and to reflect inotropic state (31-34). Because Emax can be affected by cardiac size independent of inotropic state $(35,36)$, we also evaluated contractility using Emax corrected for end-diastolic volume. Contractile function was also analyzed using the afterloadcorrected velocity of circumferential fiber shortening ( $V_{\mathrm{c}-\text {-stress rela- }}$ tionship) (21-24). This relationship has an advantage of being dimensionless and therefore is independent of cardiac size.

The $E \max$ and stress-corrected $V_{\text {cf }}$ relationships were obtained for each animal by load manipulation during a single ventricular contrast (Renographin 76) injection. Arterial pressure was reduced for $\sim 10 \mathrm{~s}$ by inflation of the vena caval balloon. The nadir of peak-systolic pressure was maintained above $60 \mathrm{mmHg}$ to assure adequate coronary perfusion (37). Simultaneous left ventricular injection and aortic manometry were carried out during subsequent balloon deflation. Balloon deflation produced a beat to beat increase in systemic pressure, yielding beat to beat load alteration. Four to six beats were analyzed per deflation. Contractile function was determined after propranolol (1 $\mathrm{mg} / \mathrm{kg}$ i.v.) administration in all of the experimental animals and before and after propranolol administration in a subgroup of six. This subgroup study was performed to test the ability of our methods to detect a change in contractile function when a known negative inotropic agent was administered. Successful beta blockade was confirmed by the absence of an increase in heart rate during isoproterenol $(1.0 \mu \mathrm{g} / \mathrm{kg}$ per min) administration, performed after all contractile studies had been completed.

Emax was determined as the slope of the pressure-volume relationship derived from all of the analyzed beats during load manipulation. A high correlation coefficient $(r \geq 0.95)$ was obtained for each animal (example, Fig. 2). Emax was corrected for end-diastolic volume $\left(E_{\max _{c}}\right)$ by multiplying $E_{\max }$ by end-diastolic volume.

The $V_{\mathrm{cr}}$-stress relationship was determined frame by frame as described under human studies. However, unlike in our patients where only one $V_{\mathrm{cf}}$-stress point was available per subject, in swine a $V_{\mathrm{cr}}$-stress point was produced for each differently loaded cardiac cycle. An exponential regression of mean stress vs. the velocity of fiber shortening was determined using data from all analyzed beats $(21,22)$ (example, Fig. 3). Values for $V_{\mathrm{cf}}$ at the common mean stress levels of 50 and 100 $\mathrm{kdyn} / \mathrm{cm}^{2}$ (in the range of the data), were calculated from this relationship.

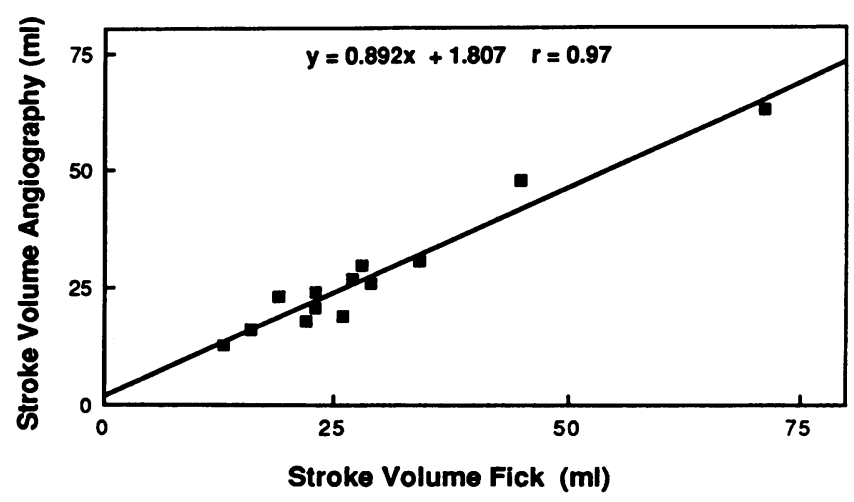

Figure 1. Angiographic versus Fick stroke volume. Angiographically determined stroke volume in swine is plotted against stroke volume determined by the Fick method. A close correlation is demonstrated.

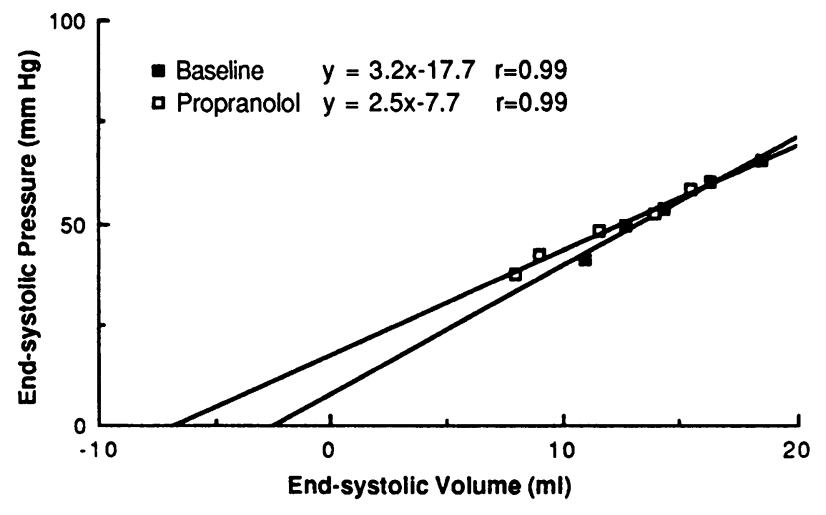

Figure 2. Emax determination. Maximum elastance (Emax) was calculated in the Yucatan swine as the slope of the end-systolic pressure-end-systolic volume relationship. Four to six beats were utilized in each analysis, and the coefficient of correlation was $\geq 0.95$ in all cases. Emax declined after propranolol administration.

Statistics. Statistical comparison of the three groups of children was performed by a one-way analysis of variance followed by a Tukey test for pairwise comparison. The exponential $V_{\mathrm{cf}}$-stress relationship and 95\% prediction band were obtained from the individual data of control children using standard statistical formulae (38). Comparison of the control swine with the VSD swine was made using a two-tailed $t$ test for independent groups. A paired $t$ test was used to compare swine evaluated before and after propranolol administration. Linear regression by the least squares method was used to develop the Emax relationship and for comparing angiographic and Fick stroke volume. Comparisons were considered statistically significant at the $P=0.05$ level. All data are presented as the mean \pm 1 standard deviation.

\section{Results}

\section{Human studies}

Hemodynamic and cineangiographic data. Cardiac catheterization data are presented in Table II. The heart rate was similar among the three groups. Angiographically determined cardiac index and forward stroke volume index (Fig. 4) were re-

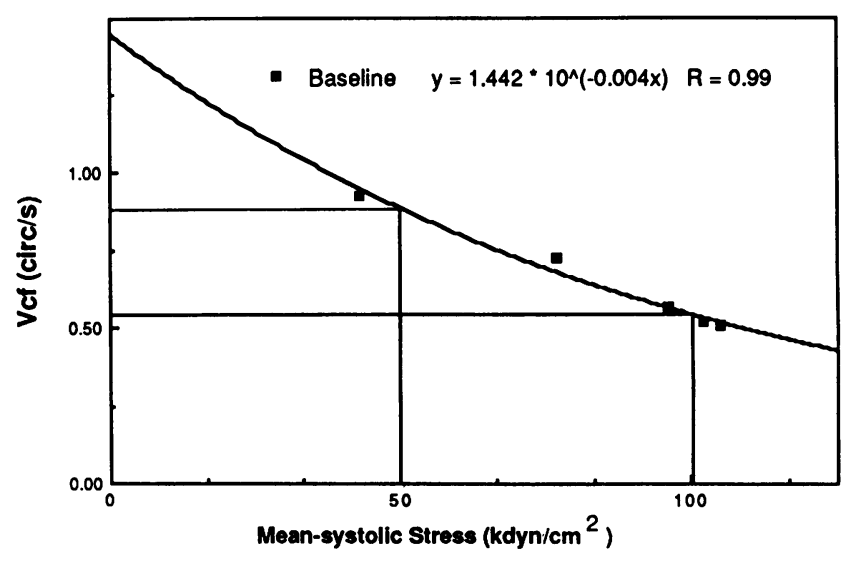

Figure 3. $V_{\mathrm{cr}}$-stress determination. Exponential regression of the mean velocity of circumferential fiber shortening $\left(V_{\mathrm{cf}}\right)$-mean systolic stress data in the baseline state (closed boxes) is presented for a Yucatan swine with a ventricular septal defect. Contractility was assessed by calculating $V_{\mathrm{cf}}$ at the common end-systolic stress of 50 and $100 \mathrm{kdyn} / \mathrm{cm}^{2}$. 
Table II. Patient Hemodynamic and Cineangiographic Data

\begin{tabular}{|c|c|c|c|}
\hline & Controls & VSD-NGD & VSD-FTT \\
\hline HR $(b p m)$ & $103 \pm 20$ & $98 \pm 13$ & $115 \pm 13$ \\
\hline $\mathrm{CI}\left(\mathrm{l} / \mathrm{min} / \mathrm{m}^{2}\right)$ & $5.0 \pm 1.4$ & $3.9 \pm 1.4$ & $3.4 \pm 0.9^{*}$ \\
\hline $\operatorname{FSVI}\left(\mathrm{ml} / \mathrm{m}^{2}\right)$ & $49 \pm 15$ & $41 \pm 16$ & $31 \pm 8^{*}$ \\
\hline TSVI $(m l / m)$ & $49 \pm 15$ & $85 \pm 23^{\ddagger}$ & $86 \pm 20^{\ddagger}$ \\
\hline QP/QS & - & $2.3 \pm 0.8$ & $2.8 \pm 0.6$ \\
\hline $\operatorname{EDVI}\left(\mathrm{ml} / \mathrm{m}^{2}\right)$ & $75 \pm 21$ & $137 \pm 34^{\ddagger}$ & $143 \pm 48^{\ddagger}$ \\
\hline $\operatorname{ESVI}\left(\mathrm{ml} / \mathrm{m}^{2}\right)$ & $25 \pm 9$ & $48 \pm 27^{*}$ & $59 \pm 28^{*}$ \\
\hline $\operatorname{LVMI}\left(\mathrm{g} / \mathrm{m}^{2}\right)$ & $73 \pm 18$ & $109 \pm 27^{\ddagger}$ & $112 \pm 22^{\ddagger}$ \\
\hline $\operatorname{RVEDP}(\mathrm{mmHg})$ & $7 \pm 3$ & $9 \pm 2$ & $9 \pm 2$ \\
\hline $\operatorname{RVSP}(m m H g)$ & $23 \pm 7$ & $33 \pm 12$ & $65 \pm 19^{\ddagger \S}$ \\
\hline LVEDP $(\mathrm{mmHg})$ & $8 \pm 3$ & $12 \pm 3^{\ddagger}$ & $12 \pm 2^{\ddagger}$ \\
\hline $\operatorname{LVSP}(m m H g)$ & $107 \pm 13$ & $95 \pm 10$ & $98 \pm 15$ \\
\hline PAP $(m m H g)$ & $15 \pm 5$ & $22 \pm 8$ & $46 \pm 13^{\ddagger \S}$ \\
\hline PVRI $\left(d y n / s / \mathrm{cm}^{-5} / \mathrm{m}^{2}\right)$ & $118 \pm 70$ & $287 \pm 204$ & $754 \pm 315^{\ddagger \S}$ \\
\hline SVRI $\left(d y n / s / \mathrm{cm}^{-5} / \mathrm{m}^{2}\right)$ & $1,386 \pm 626$ & $1,519 \pm 428$ & $1,634 \pm 576$ \\
\hline $\operatorname{EDS}\left(k d y n / \mathrm{cm}^{2}\right)$ & $29 \pm 8$ & $45 \pm 15^{\ddagger}$ & $47 \pm 12^{\ddagger}$ \\
\hline $\operatorname{ESS}\left(k d y n / c^{2}\right)$ & $127 \pm 30$ & $136 \pm 45$ & $143 \pm 60$ \\
\hline PSS $\left(k d y n / \mathrm{cm}^{2}\right)$ & $303 \pm 66$ & $295 \pm 62$ & $305 \pm 74$ \\
\hline $\operatorname{MSS}\left(k d y n / \mathrm{cm}^{2}\right)$ & $213 \pm 41$ & $222 \pm 45$ & $221 \pm 66$ \\
\hline EF & $0.66 \pm 0.07$ & $0.65 \pm 0.08$ & $0.62 \pm 0.09$ \\
\hline$V_{\mathrm{cf}}(c / s)$ & $1.18 \pm 0.19$ & $1.17 \pm 0.25$ & $1.13 \pm 0.18$ \\
\hline
\end{tabular}

HR, heart rate; CI, systemic cardiac index; FSVI, forward stroke volume index; $\mathrm{QP} / \mathrm{QS}$, ratio of pulmonary to systemic blood flow; EDVI, end-diastolic volume index; ESVI, end-systolic volume index; EF, ejection fraction; ESS, end-systolic stress; EDS, end-diastolic stress; LVMI, left ventricular muscle mass index; PAP, mean pulmonary artery pressure; TSVI, total stroke volume index; RVEDP, right ventricular end-diastolic pressure; RVSP, right ventricular peak systolic pressure; LVEDP, left ventricular end-diastolic pressure; LVSP, left ventricular peak systolic pressure; PVRI, pulmonary vascular resistance index; SVRI, systemic vascular resistance index; PSS, peak systolic stress; MSS, mean-systolic stress; ${ }^{*} P<0.05$ vs. controls; ${ }^{\ddagger} P$ $<0.01$ vs. controls; ${ }^{\S} P<0.01$ vs. VSD-NGD. Absence of a sign indicates no statistically significant difference was present.

duced in group VSD-FTT $\left(31 \pm 8 \mathrm{ml} / \mathrm{m}^{2}\right)$ compared to normal (NL) subjects $\left(49 \pm 15 \mathrm{ml} / \mathrm{m}^{2}, P<0.05\right)$, while forward stroke volume in VSD-NGD $41 \pm 16 \mathrm{ml} / \mathrm{m}^{2}$ was similar to group NL. The shunt ratio was somewhat larger in the VSD-FTT group but this difference did not reach statistical significance. The end-diastolic volume index was significantly increased in VSD-NGD $\left(137 \pm 34 \mathrm{ml} / \mathrm{m}^{2}\right)$ and in VSD-FTT $(143 \pm 48$ $\left.\mathrm{ml} / \mathrm{m}^{2}\right)$ compared to NL $\left(75 \pm 21 \mathrm{ml} / \mathrm{m}^{2}, P<0.01\right)$. End-systolic volume was increased in both groups with VSD compared to NL $(P<0.05)$. Ejection fraction, $V_{\mathrm{cf}}$, end-systolic

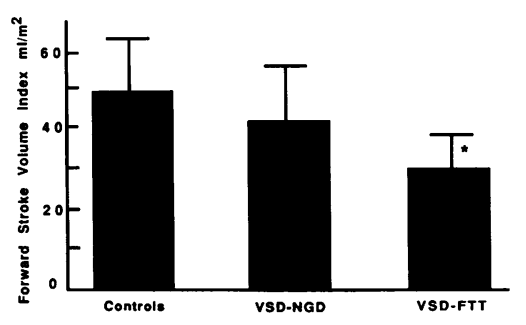

Figure 4. Forward stroke volume index. Forward stroke volume even after indexing for body surface area was reduced in children with VSD and FTT, compared to those with VSD and NGD and controls $(P<0.05)$.

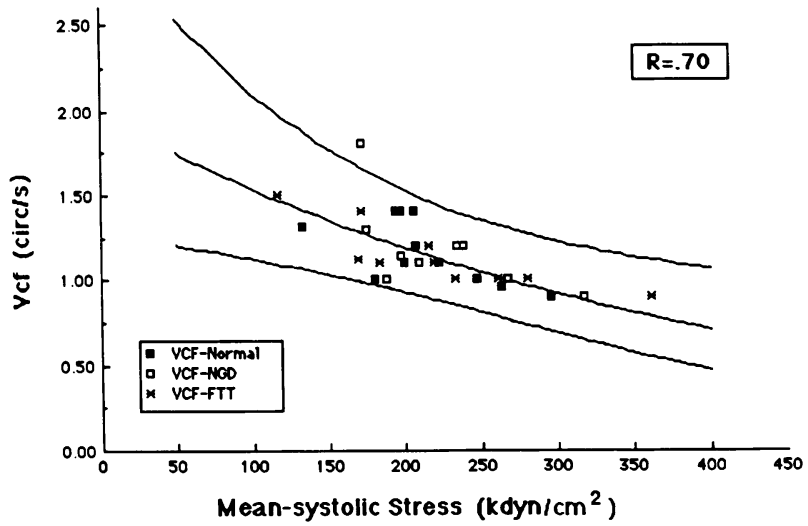

Figure $5 . V_{\mathrm{cr}}$-stress in children. The $V_{\mathrm{cf}}$ mean-systolic stress (MSS) relationship is presented for normal subjects (closed boxes), children with VSD and NGD (VSD-NGD, open boxes) and children with VSD and failure to thrive (VSD-FTT, stars). The exponential regression function derived from the individual control data is indicated with its $95 \%$ prediction band. There was nearly complete overlap of the data, and all but one patient from all three groups fell within the 95\% prediction band. This finding suggests that contractile function is normal in these patients with VSD.

stress, peak-systolic stress and mean-systolic stress were similar among the three groups. End diastolic stress was elevated in VSD-FTT $\left(47 \pm 19 \mathrm{kdyn} / \mathrm{cm}^{2}\right)$ and VSD-NGD $(45 \pm 15 \mathrm{kdyn} /$ $\left.\mathrm{cm}^{2}\right)$ compared to NL $\left(29 \pm 8 \mathrm{kdyn} / \mathrm{cm}^{2}, P<0.01\right)$. The left ventricular muscle mass index was increased in both VSD groups compared to NL $(P<0.01)$. The pulmonary artery pressure was increased in VSD-FTT compared to the other groups $(P<0.01)$.

The mean velocity of circumferential fiber shortening, end-systolic stress relationship is presented in Fig. 5. Individual data from each of the patients and the exponential regression relationship derived from the normal subjects are shown. There is nearly complete overlap of patient data from each group, and all VSD patients fell within the $95 \%$ prediction band for normal subjects except for one patient who fell outside up and to the left suggesting that contractile function was increased.

Postoperative clinical data. Followup data from ambulatory clinic records were available for all but two children (one from each VSD group) and are presented in Table III. All children with FTT underwent operation, one of whom died

Table III. Postoperative Clinical Data

\begin{tabular}{lcc}
\hline & VSD-NGD & VSD-FTT \\
\hline Age at followup $(y r)$ & $5.9 \pm 3.5$ & $3.3 \pm 2.1$ \\
Followup after operation $(m o)$ & $15 \pm 8$ & $12 \pm 9$ \\
Postop weight gain $(k g)$ & $4.9 \pm 2.4$ & $4.4 \pm 1.9$ \\
Postop weight increase compared to & & \\
$\quad$ predicted $(\%)$ & $121 \pm 32$ & $158 \pm 62$ \\
Postop weight percentile & $60 \pm 20$ & $20 \pm 20^{\ddagger}$ \\
& $(45 \pm 20)$ & $(<3)$ \\
\hline
\end{tabular}

${ }^{*} P<0.001$ VSD-NGD vs. VSD-FTT; ${ }^{\ddagger} P<0.01$ vs. preoperative VSD-FTT. Preoperative weight percentiles are indicated in parentheses. 
during surgery. 7 of 9 NGD patients had repair of the VSD. A minimal residual postoperative defect remained in 3 of 11 FTT patients and in 2 of the 7 NGD children who underwent operation (NS). Postoperative followup was similar for patients in both groups. Absolute postoperative weight gain and weight gain as a proportion of that predicted for the patient's age, were similar in the two groups. Postoperatively, the mean weight of group VSD-NGD continued to exceed that for group VSD-FTT $(P<0.05)$. However, the postoperative weight percentile increased significantly in group VSD-FTT following surgical repair, but did not in group VSD-NGD. In addition, 9 of the 11 children preoperatively classified as failure to thrive were classified as having normal growth and development at the time of clinical followup.

Yucatan swine data. The data obtained at cardiac catheterization of the Yucatan micro swine are presented in Table IV. VSD swine were older than their controls $(P<0.05)$, but the two groups did not differ in their body weight. Thus, it took the VSD swine longer to achieve the same weight as controls.

Table IV. Yucatan Swine Data

\begin{tabular}{|c|c|c|}
\hline & Controls & VSD \\
\hline $\mathrm{QP} / \mathrm{QS}$ & - & $2.3: 1 \pm 1.1$ \\
\hline Age $(w k)$ & $21 \pm 1$ & $27 \pm 4^{*}$ \\
\hline $\mathrm{Wt}(\mathrm{kg})$ & $21 \pm 2$ & $25 \pm 7$ \\
\hline $\mathrm{HR}(b p m)$ & $116 \pm 13$ & $122 \pm 21$ \\
\hline LVMI $(g / k g)$ & $2.4 \pm 0.4$ & $2.9 \pm 0.8$ \\
\hline $\operatorname{EDVI}(m l / k g)$ & $1.9 \pm 0.5$ & $2.2 \pm 0.8$ \\
\hline $\mathrm{ESVI}(\mathrm{ml} / \mathrm{kg})$ & $0.8 \pm 0.3$ & $0.9 \pm 0.4$ \\
\hline $\mathrm{EF}$ & $0.58 \pm 0.08$ & $0.57 \pm 0.08$ \\
\hline IP $(m m H g)$ & $81 \pm 8$ & $73 \pm 11$ \\
\hline PAP $(m m H g)$ & $21 \pm 10$ & $20 \pm 6$ \\
\hline $\mathrm{PCW}(m m H g)$ & $4 \pm 4$ & $9 \pm 5$ \\
\hline $\mathrm{VO}_{2}(\mathrm{ml} / \mathrm{m} / \mathrm{kg})$ & $2.6 \pm 0.3$ & $2.8 \pm 0.2$ \\
\hline FSVI $(m l / k g)$ & $1.1 \pm 0.26$ & $0.65 \pm 0.11^{\ddagger}$ \\
\hline $\mathrm{CI}($ liters/min/kg) & $0.12 \pm 0.03$ & $0.08 \pm 0.01^{\ddagger}$ \\
\hline $\operatorname{SVRI}\left(d y n \cdot \mathrm{s} / \mathrm{cm}^{5} \cdot \mathrm{kg}\right)$ & $152 \pm 71$ & $127 \pm 67$ \\
\hline PVRI $\left(d y n \cdot s / \mathrm{cm}^{5} \cdot \mathrm{kg}\right)$ & $37 \pm 17$ & $11 \pm 5^{\S}$ \\
\hline $\operatorname{EDS}\left(d y n / \mathrm{cm}^{2}\right)$ & $9 \pm 5$ & $22 \pm 11^{*}$ \\
\hline $\operatorname{ESS}\left(d y n / \mathrm{cm}^{2}\right)$ & $111 \pm 28$ & $117 \pm 36$ \\
\hline$E \max$ & $2.2 \pm 0.7$ & $1.5 \pm 0.8$ \\
\hline $\operatorname{Emax}_{\mathrm{c}}$ & $84 \pm 27$ & $68 \pm 30$ \\
\hline$V_{\text {crso }}(\operatorname{circ} / s)$ & $1.04 \pm 0.14$ & $0.99 \pm 0.15$ \\
\hline$V_{\mathrm{cf} 100}(\mathrm{circ} / \mathrm{s})$ & $0.68 \pm 0.14$ & $0.70 \pm 0.13$ \\
\hline
\end{tabular}

QP/QS, ratio of pulmonary to systemic blood flow; Wt, body weight; HR, heart rate; LVMI, left ventricular muscle mass index; EDVI, end-diastolic volume index; ESVI, end-systolic volume index; EF, ejection fraction; IP, incisural pressure; PAP, mean pulmonary artery pressure; $\mathrm{PCW}$, pulmonary capillary wedge pressure; $\mathrm{VO}_{2}$, oxygen consumption; FSVI, forward stroke volume index; CI, systemic cardiac index; SVRI, systemic vascular resistance index; PVRI, pulmonary vascular resistance index; EDS, end-diastolic stress; ESS, endsystolic stress; Emax, maximum systolic elastance; $E_{\max _{c}, E \max }$ corrected for end-diastolic volume; $V_{\text {crso }}, V_{\text {cfl } 100}$, mean velocity of circumferential fiber shortening at a common end-systolic stress of 50 and $100 \mathrm{kdyn} / \mathrm{cm}^{2}$.

${ }^{*} P<0.05$ vs. controls; ${ }^{\ddagger} P<0.001$ vs. controls; ${ }^{\S} P<0.01$ vs. controls. Absence of a sign indicates no statistically significant difference was present.
Heart rate, left ventricular muscle mass index, end-systolic volume index and ejection fraction were also similar in the two groups. Incisural pressure, mean pulmonary artery pressure and pulmonary capillary wedge pressure and $\mathrm{A}-\mathrm{VO}_{2}$ difference were also similar in the two groups. VSD animals had a significantly reduced forward stroke volume index $(0.65 \pm 0.11 \mathrm{vs}$. $1.1 \pm 0.26 \mathrm{ml} / \mathrm{kg}, P<0.001)$ and systemic cardiac index $(0.08 \pm 0.01$ vs. $0.12 \pm 0.031 / \mathrm{kg}, P<0.001)$. While systemic vascular resistance index was similar, pulmonary vascular resistance index was significantly reduced $(P<0.01)$ in swine with VSD. End-diastolic stress was elevated in the group with VSD $\left(22 \pm 11\right.$ vs. $\left.9 \pm 5 \mathrm{kdyn} / \mathrm{cm}^{2}, P<0.05\right)$ but end-diastolic volume index was similar in the two groups $-1.9 \pm 0.5 \mathrm{~cm}^{3} / \mathrm{kg}$ normal, vs. $2.2 \pm 0.8 \mathrm{~cm}^{3} / \mathrm{kg}$ VSD. End-systolic stress was similar in the two groups.

Indices of contractile function demonstrated no difference between the VSD swine and controls. $E \max (1.5 \pm 0.8$, VSD vs. $2.2 \pm 0.7$, Controls) and $E \max _{c}(68 \pm 30$, VSD vs. $84 \pm 27$, controls) were similar, as were $V_{\text {cf }}$ at the common mean-systolic stress levels of $50(0.99 \pm 0.15 \mathrm{circ} / \mathrm{s}, \mathrm{VSD}$ vs. $1.04 \pm 0.14 \mathrm{circ} / \mathrm{s}$, controls) and $100(0.70 \pm 0.13 \mathrm{circ} / \mathrm{s}$, VSD vs. $0.68 \pm 0.14 \mathrm{circ} / \mathrm{s}$, controls) $\mathrm{kydn} / \mathrm{cm}^{2}$ (Fig. 6).

Contractile function data in the subgroup of six animals studied before and after administration of propranolol demonstrated a reduction in $E \max , V_{\mathrm{cfs} 0}$ and $V_{\mathrm{cf} 100}$ after institution of beta blockade: $E$ max decreased from $2.8 \pm 0.4$ to $2.1 \pm 0.5$ ( $P$ $<0.01), V_{\text {cf5o }}$ fell from $0.95 \pm 0.12$ to $0.75 \pm 0.09(P<0.05)$ and $V_{\text {cfloo }}$ fell from $0.72 \pm 0.15$ to $0.54 \pm 0.09(P<0.001)$.

\section{Discussion}

Our study confirmed that VSD complicated by FTT is associated with decreased systemic cardiac output and forward stroke volume. Although previous investigators have suggested that FTT in VSD is due to decreased caloric intake (7), increased metabolic rate (10), or decreased absorption (4), Miller et al. (8) found decreasing systemic blood flow with increasing shunt ratio and suggested a cardiac cause in patients with VSD and FTT. They also described an inverse relationship between weight percentile for age and shunt ratio. Levy et al. (9) also

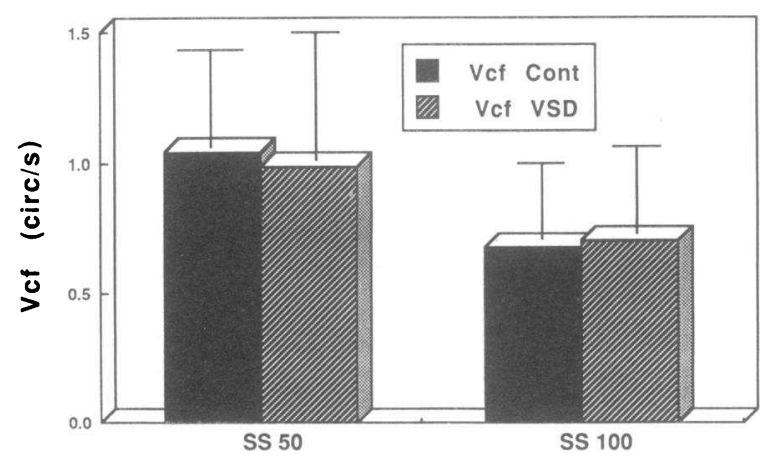

Figure 6. $V_{\mathrm{cr}}$-stress in swine. The mean velocity of circumferential fiber shortening $\left(V_{\mathrm{cf}}\right)$ at the common mean-systolic stress levels of 50 (SS 50) and 100 (SS 100) $\mathrm{kdyn} / \mathrm{cm}^{2}$ following beta blockade are shown for Yucatan micro swine with congenital ventricular septal defects (striped histogram) and for controls (black histograms). Velocity of shortening was similar at both levels of stress, suggesting similar contractility in the two groups. 
found that growth was inversely related to the shunt ratio in VSD. Further support for a direct cardiac cause of FTT in VSD is given by studies which show a return to normal growth and development after the cardiac defect is repaired (11-15).

Our study was designed to determine the mechanism of reduced forward cardiac output in VSD-FTT. At a given heart rate, systemic cardiac output is determined by forward stroke volume. Forward stroke volume in VSD is a function of shunt size, myocardial contractility, afterload, preload, and ventricular volume. We examined each of these hemodynamic factors to assess their contribution to the diminished forward flow observed.

Contractile function. An important finding in our study was that contractile function was normal in subjects with VSD and FTT. Myocardial contractile function was assessed using ejection fraction, uncorrected mean velocity of circumferential fiber shortening $\left(V_{\mathrm{cf}}\right)$ and the stress-velocity relationship. Ejection fraction was similar in the three groups of children. Ejection fraction is however both preload and afterload dependent (39). The uncorrected $V_{\text {cf }}$, a preload independent index of contractility (25) was also similar in the three groups but this index varies inversely with afterload (23). Correction of $V_{\text {cf }}$ for afterload (systolic stress) yields a relatively load independent measure of contractile function (23). Stress-corrected $V_{\text {cf }}$ (Fig. 5) was similar in the controls and in children with VSD. These data suggest that contractile function was normal in our children with VSD with or without FTT and was not the cause of the reduced stroke volume found in VSD-FTT group.

Relationship to other studies of contractile function. Others have suggested that contractile function is reduced postoperatively in patients with VSD. One year after operation, Maron et al. (40) found a reduced cardiac output during exercise in postoperative VSD patients compared to nonoperated controls. A reduced inotropic reserve was suggested as the cause. Jarmakani et al. (41) studied postoperative VSD patients and compared their results to those of a non-operated normal group. Resting ejection fraction and $V \max$ were decreased in the VSD group. However the catheterization of the VSD patients in this study was performed under halothane anesthesia, a known negative inotrope. Jablonsky et al. (42) found that postoperative VSD patients demonstrated a decreased resting ejection fraction and a blunted increase of ejection fraction during exercise compared to their controls. In all of the above studies however, the controls were a nonoperated group and thus it is not clear whether the observed abnormalities in cardiac performance were due to the VSD or surgery. In contrast our study is in agreement with several recent studies (43-45) which found normal ventricular function in VSD patients compared to controls. It is likely that the unavailability of intraoperative myocardial preservation techniques affected the results of the earlier investigations.

To help resolve the above conflicting reports and because adrenergic support could have concealed an inotropic deficit in our patients, we further studied contractile function in an experimental animal model of congenital VSD in which stroke volume was also diminished and in which load manipulation and beta blockade could be ethically performed. Load manipulation allowed us to generate the peak elastance $(E \max )$, volume-corrected peak elastance $\left(E \max _{c}\right)$ and stress-corrected $V_{\text {ef }}$ relationships, which have been used in previous studies to assess contractile function (21-24, 31-36). Evaluation of contractile function was performed during one ventricular con- trast injection avoiding the use of pharmacologic agents to manipulate load which might by themselves have affected contractile function. The adequacy of beta blockade was confirmed by the absence of a chronotropic response to administration in pharmacologic doses of the beta agonist isoproterenol. The results in swine, like those in children indicate no reduction in contractile function as the cause of the reduced stroke volume which we observed.

To assess the sensitivity of our contractile indices to changes in function, we compared $E \max , V_{\text {cfso }}$ and $V_{\text {crioo }}$ before and after propranolol administration. Emax, and velocity of circumferential shortening at both levels of mean-systolic stress, declined after propranolol administration. Thus, our methods correctly demonstrated the reduction in contractile function produced by a known negative inotropic drug. Therefore the absence of a difference between VSD and controls was probably not due to an inability to discern changes in function had any been present.

Afterload. Afterload, assessed as the end-systolic stress, peak-systolic stress or mean systolic stress was similar in the three groups of children and in both groups of swine. In addition, calculated systemic vascular resistance in swine was similar in the control and VSD groups. Thus the reduced systemic cardiac output was not likely due to increased afterload.

Preload. Preload was evaluated as end-diastolic pressure and end-diastolic stress. Both were increased in both groups of VSD children compared to normals. At the myocardial level, preload is the extent of stretch of individual sarcomeres. We were not able to evaluate sarcomere length in our group of patients or cohort of swine. However, that end-diastolic stress was increased in the group of children with VSD and FTT, and in swine suggests that reduced preload was not responsible for the diminished forward stroke volume found in these subjects.

End-diastolic volume and shunt size. When forward stroke volume is reduced by an intracardiac shunt, systemic flow can be augmented by ventricular enlargement. Both groups of children with VSD exhibited such an increase in end-diastolic volume. However, shunt size was somewhat larger $(21 \%)$ in VSD-FTT than VSD-NGD. Although this difference was not statistically significant, it may have been "clinically" significant. The $21 \%$ larger shunt size was coincident with the $25 \%$ fall reduction in forward stroke volume, which was statistically significant. In the absence of reduced contractile function, reduced preload or increased afterload, it is possible that it was end-diastolic volume which, although increased, was not increased enough to maintain normal forward stroke volume in the face of possibly larger shunts in the children with FTT. This argument is further strengthened by the finding that a similar inadequate (and insignificant) increase in end-diastolic volume occurred in swine.

The failure of the left ventricle to sufficiently enlarge in children with FTT is an observation in clear contrast to other chronic volume overload states. In the adult with compensated chronic mitral regurgitation or aortic insufficiency, adequate eccentric hypertrophy occurs to maintain forward stroke output $(46,47)$. Grossman has hypothesized that increased enddiastolic stress is the major stimulus to eccentric hypertrophy and cavity dilatation. However in both VSD groups, end-diastolic stress was increased. It is possible that some theoretic limit to the extent of hypertrophy prevented further increases in end-diastolic volume needed to compensate the somewhat large shunts in the VSD-FTT children. 
Limitations. The validity of our results suggesting that forward stroke volume is reduced and that end-diastolic volume is not appropriately augmented in children with VSD and FTT, rests upon our ability to accurately measure ventricular volume by standard angiographic techniques. Although these techniques have known limitations (48), the identical techniques were applied to all three groups of children thus avoiding introduction of a systematic error affecting one group. Our angiographic measurements were validated in the swine model where systemic cardiac output and stroke volume measured independently by ventriculography and by the Fick method were similar. The close relationship between cardiac output and stroke volume derived from these two techniques, helps confirm the validity of our measurements and our conclusions that VSD-FTT is associated with reduced forward stroke volume. Our pressures were recorded just before, but not simultaneously with, angiography in our children. While this could have introduced error, there was no evidence that the hemodynamics changed in this short interval between recording and injection. In the swine, pressure recording and angiography were simultaneous.

We utilized isoflurane, a negative inotropic agent during cardiac catheterization in our cohort of swine. Differential use of the drug between controls and VSD animals could have altered our conclusions regarding contractile function. However, isoflurane was used at the lowest concentration permitting catheterization, and within a narrow dose range that did not differ between swine with VSD and controls; both groups of swine received a similar dose of anesthesia. Therefore, it is unlikely that our use of anesthesia differentially altered contractile function in controls versus the VSD group of swine.

Conclusion. In the presence of a congenital VSD, FTT is commonly observed. In this study, FTT was associated with a decreased systemic output and forward stroke volume. We conclude that reduced stroke volume was not due to impaired contractile function, excess afterload, or reduced preload. The exact mechanism for the reduced stroke volume remains speculative, however, it probably occurred secondary to a failure of end-diastolic volume to increase adequately despite the presence of an increased end-diastolic stress.

\section{Acknowledgments}

The authors wish to thank Linda Paddock for her secretarial assistance. Supported by the Gazes Cardiac Research Institute, Charles River Foundation, and MUSC Institutional grant funds.

\section{References}

1. Bloomfield, D. K. 1964. The natural history of ventricular septal defect in patients surviving infancy. Circulation. 29:914-955.

2. Selzer, A. 1949. Defect of the ventricular septum: summary of the twelve cases and review of the literature. Arch. Intern. Med. 84:798-823.

3. Fyler, D. C., A. M. Rudolph, M. H. Wittenborg, and A. S. Nadas. 1958. Ventricular septal defect in infants and children: a correlation of clinical, physiologic and autopsy data. Circulation. 18:833-851.

4. Markiewicz, A., D. Wojczuk, and W. Iljin. 1976. Xylose absorption before and after surgical correction of atrial septal defect (ASD) and ventricular septal defect (VSD). Eur. J. Pediatr. 124:57-63.

5. Owen-Thomas, J. B., F. Meade, R. S. Jones, and G. Jackson Rees. 1971. The measurement of oxygen uptake in infants with congenital heart disease during general anaesthesia and intermittent positive pressure ventilation. Br. J. Anaesth. 43:746-752.
6. Pittman, J. G., and P. Cohen. 1964. The pathogenesis of cardiac cachexia. N. Engl. J. Med. 271:403-409.

7. Menon, G., and E. M. E. Poskitt. 1985. Why does congenital heart disease cause failure to thrive? Arch. Dis. Child. 60:1134-1139.

8. Miller, R. H., G. L. Schiebler, P. Grumbar, and L. J. Krovetz. 1969. Relation of hemodynamics to height and weight percentiles in children with ventricular septal defects. Am. Heart J. 78:523-529.

9. Levy, R. J., A. Rosenthal, O. S. Miettinen, and A. S. Nadas. 1978. Determinants of growth in patients with ventricular septal defect. Circulation. 57:793-797.

10. Stocker, F. P., W. Wilkoff, O. S. Miettinen, and A. S. Nadas. 1972. Oxygen consumption in infants with heart disease. Relationship to severity of congestive failure, relative weight, and caloric intake. $J$. Pediatr. 80:43-51.

11. Yeager, S. B., M. D. Freed, J. F. Keane, W. I. Norwood, and A. R. Castaneda. 1984. Primary surgical closure of ventricular septal defect in the first year of life results in 128 infant. J. Am. Coll. Cardiol. 3:1269-1276.

12. Rein, J. G., M. D. Freed, W. I. Norwood, and A. R. Castaneda. 1977. Early and late results of closure of ventricular septal defect in infancy. Ann. Thorac. Surg. 24:19-27.

13. Rosenthal, A., and A. R. Castaneda. 1975. Growth and development after cardiovascular surgery in infants and children. Prog. Cardiovasc. Dis. 18:27-37.

14. Sigmann, J. M., B. L. Perry, D. M. Gehrendt, A. M. Stern, M. M. Kirsh, and H. E. Sloan. 1977. Ventricular septal defect. Results after repair in infancy. Am. J. Cardiol. 39:66-71.

15. Sigmann, J. M., A. M. Stern, and H. E. Sloan. 1967. Early surgical correction of large ventricular septal defects. Pediatrics. 39:4-13.

16. Nelson, W. E., R. E. Behrman, and V. C. Vaughan, III. 1983. Textbook of Pediatrics. W. B. Saunders, Philadelphia.

17. Kennedy, J. W., S. E. Trenholme, and I. S. Kasser. 1970. Left ventricular volume and mass from single-plane cineangiocardiogram. A comparison of antero-posterior and right anterior oblique methods. Am. Heart J. 80:343-352.

18. Hugenholtz, P. A., E. Kaplan, and E. Hull. 1969. Determination of left ventricular wall thickness of angiocardiography. Am. Heart J. 78:513-522.

19. Mirsky, I. 1969. Left ventricular stresses in the intact human heart. Biophys. J. 9:189-208.

20. Rackley, C. E., H. T. Dodge, Y. D. Coble, Jr., and R. E. Hay. 1964. A method for determining left ventricular mass in man. Circulation. 29:666-671.

21. Ross, J., Jr. 1976. Afterload mismatch and preload reserve: a conceptual framework for analysis of ventricular function. Prog. Cardiovasc. Dis. 18:255-264.

22. Gunther, S., and W. Grossman. 1979. Determinants of ventricular function in pressure-overload hypertrophy in man. Circulation. 59:679-688.

23. Colan, S. D., K. M. Borow, and A. Neumann. 1984. Left ventricular end-systolic wall stress-velocity of fiber shortening relation: a load independent index of myocardial contractility. J. Am. Coll. Cardiol. 4:715-724.

24. Carabello, B. A., and L. Gittens. 1987. Cardiac mechanics and function in obese normotensive persons with normal coronary arteries. Am. J. Cardiol. 59:469-473.

25. Nixon, J. V., R. G. Murray, P. D. Leonard, J. H. Mitchell, and C. G. Blomqvist. 1982. Effect of large variations in preload on left ventricular performance characteristics in normal subjects. Circulation. 65:698-703.

26. Lynch, C., III. 1986. Differential depression of myocardial contractility by halothane and isoflurane in vitro. Anesthesiology. 64:620-631.

27. Philbin, D. M., and E. Lowenstein. 1975. Hemodynamic consequences of the combination of isoflurane anesthesia (1 MAC) and beta-adrenergic blockade in the dog. Anesthesiology. 42:567-573. 
28. Merin, R. G. 1981. Are the myocardial functional and metabolic effects of isoflurane really different from those of halothane and enflurane? Anesthesiology. 55:398-408.

29. Eger, E. I., II. 1981. Isoflurane: A review. Anesthesiology. 55:559-576.

30. Schieber, R. A., A. Namnoum, A. Sugden, G. K. Shiu, R. A. Orr, and D. R. Cook. 1986. Hemodynamic effects of isoflurane in the newborn piglet: comparison with halothane. Anesth. Analg. 65:633638.

31. Suga, H., K. Sagawa, and A. A. Shoukas. 1973. Load independence of the instantaneous pressure-volume ratio of the canine left ventricle and effect of epinephrine and heart rate on the ratio. Circ. Res. 32:314-322.

32. Sagawa, K., H. Suga, A. A. Shoukas, and K. M. Bakalar. 1977. End-systolic pressure/volume ratio: a new index of ventricular contractility. Am. J. Cardiol. 40:748-753.

33. Grossman, W., E. Braunwald, T. Mann, L. P. McLaurin, and L. H. Green. 1977. Contractile state of the left ventricle in man as evaluated from end-systolic pressure-volume relations. Circulation. $56: 845-852$.

34. Weber, K. T., and J. S. Janicki. 1977. Instantaneous force-velocity-length relations: experimental findings and clinical correlates. Am. J. Cardiol. 40:740-747.

35. Suga, H., R. Hisano, Y. Goto, and O. Yamada. 1984. Normalization of end-systolic pressure volume relation and Emax of different sized hearts. JPN. Circulation J. 48:136-143.

36. Belcher, P., L. E. Boerboom, and G. N. Olinger. 1985. Standarization of end-systolic pressure-volume relation in the dog. Am. J. Physiol. 249:H547-553.

37. Rouleau, J., L. E. Boerboom, A. Surjadhana, and J. I. E. Hoffman. 1979. The role of autoregulation and tissue diastolic pressures in the transmural distribution of left ventricular blood flow in anesthetized dogs. Circ. Res. 45:804-815.

38. Kleinbaum, D. G., and L. L. Kupper. 1978. Applied Regression Analysis and Other Multivariate Methods. Duxbury Press, Boston. 50-58.
39. Quinones, M. A., W. H. Gaasch, and J. K. Alexander. 1976. Influence of acute changes in preload, afterload, contractile state and heart rate on ejection phase and isovolumic indices of myocardial contractility in man. Circulation. 53:293-302.

40. Maron, B. J., D. R. Redwood, J. W. Hirshfeld, Jr., R. E. Goldstein, A. G. Morrow, and S. E. Epstein. 1973. Postoperative assessment of patients with ventricular septal defect and pulmonary hypertension: response to intense upright exercise. Circulation. 48:864-874.

41. Jarmakani, J. M., T. P. Graham, Jr., and R. V. Canent, Jr. 1972. Left ventricular contractile state in children with successfully corrected ventricular septal defect. Circulation. 45(Suppl. I):102-110.

42. Jablonsky, G., J. D. Hilton, P. P. Liu, J. E. Morch, M. N. Druck, B.-Z. Bar-Shlomo, and P. R. McLaughlin. 1983. Rest and exercise ventricular function in adults with congenital ventricular septal defects. Am. J. Cardiol. 51:293-298.

43. Ahmand, M., and K. A. Hallidie-Smith. 1979. Assessment of left-to-right shunt and left ventricular function in isolated ventricular septal defect. Echocardiographic study. Br. Heart J. 41:147-158.

44. Borow, K. M., J. F. Keane, A. R. Castaneda, and M. D. Freed. 1981. Systemic ventricular function in patients with tetralogy of fallot, ventricular septal defect and transposition of the great arteries repaired during infancy. Circulation. 64:878-885.

45. Sunakawa, A., Y. Nakamura, T. Shinohara, T. Yokoyama, O. Hidetaka, J. Kawai, and H. Shirotani. 1983. Angiocardiographic evaluation of ventricular septal defect. Pre- and post-operative cardiac volume characteristics. JPN Circulation J. 47:625-634.

46. Dodge, H. T., J. W. Kennedy, and J. L. Petersen. 1973. Quantitative angiographic methods in the evaluation of valvular heart disease. Prog. Cardiovasc. Dis. 16:1-23.

47. Grossman, W., D. Jones, and L. P. McLaurin. 1974. Wall stress and patterns of hypertrophy in the human left ventricle. J. Clin. Invest. 56:56-64.

48. Graham, T. P., Jr., J. M. Jarmakani, R. V. Canent, Jr., and M. N. Morrow. 1971. Left heart volume estimation in infancy and childhood. Reevaluation of methodology and normal values. Circulation. 43:895-904. 